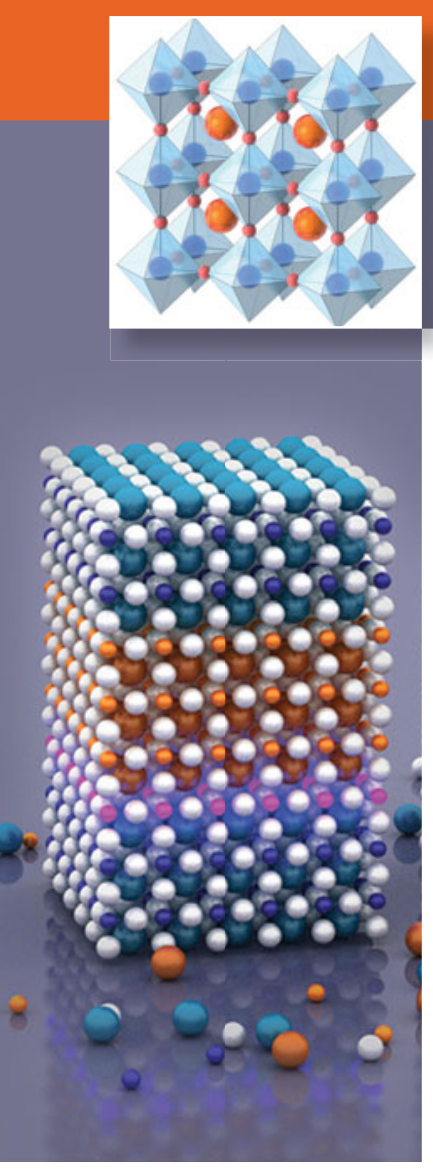

\title{
Functional oxide interfaces
}

\author{
Fabio Miletto Granozio, Gertjan Koster, and Guus Rijnders, \\ Guest Editors
}

\begin{abstract}
Functional perovskite oxides are recognized for their stunningly rich physics and for their potential as next-generation electronic materials. Their properties include high $T_{\mathrm{c}}$ superconductivity, colossal magnetoresistance, record-high dielectric/ferroelectric/piezoelectric performances, multiferroic behavior, resistive switching behavior, giant thermoelectric and magnetocaloric effects, giant ionic conduction, and catalytic behavior. Due to their intrinsic chemical and crystal similarities, functional oxides can be stacked in multilayer heterostructures exhibiting an astonishing degree of epitaxial perfection. Such artificial systems not only allow one to combine in a single device the functionalities of their individual layers, but often reveal an even wider range of emergent novel properties that can be surprisingly different from those of the single building blocks. The goal of this issue of MRS Bulletin is to present the state of the art of oxide interfaces in inscience and technology. Here we provide an introduction to their properties, serving as a base for the following topical articles.
\end{abstract}

\section{Introduction}

Functional oxides form a vast and highly diverse family of compounds recognized for their stunningly rich physics and for their potential as next-generation nano-, micro-, and macroelectronic materials. They are potential candidates to partially replace silicon and introduce complexity in solidstate technologies, including digital information and communication technologies, microactuation/microsensing, and energy conversion.

Silicon-based technologies have driven the electronics technological race during the last half-century. Silicon can be synthesized and manufactured at reduced cost, is abundant in nature, is well understood, simple, and versatile. It possesses remarkable functional properties both in its pure elemental form (e.g., $p$ - $n$-dopable semiconductor for metallic channels and junctions) and in its oxide form (e.g., wide-bandgap barrier, piezoelectric). Silicon technology benefits today from decades of previous improvements and investment. However, in spite of the extraordinary success of this material, international technology roadmaps point to qualitatively new concepts and functionalities that the "simple" physics and chemistry of $\mathrm{Si}$ cannot support. ${ }^{1}$

Research on functional oxides was boosted in the last 25 years by a number of successive discoveries for this class of materials, including high temperature superconductivity, colossal magnetoresistance, half-metallic behavior, record-high dielectric/ferroelectric/piezoelectric performances, multiferroic behavior, ${ }^{2,3}$ resistive switching behavior, giant thermoelectric and magnetocaloric effects, giant photoconductivity effects, ${ }^{4}$ giant ionic conduction, catalytic properties (including water splitting), field-induced Mott transitions, and topologically nontrivial behavior. ${ }^{5,6}$ Such discoveries have attracted a large number of scientists from different disciplines to this field. Mastering the complexity of this class of compounds offers virtually unlimited degrees of freedom in manipulating the states of matter and engineering functional properties for novel device concepts. The interfaces formed in oxide thin-film heterostructures represent a special field of research, which is the topic of this issue.

\section{Oxide interfaces}

Interfaces have, by definition, reduced dimensionality and broken inversion symmetry along their normal direction. Neighboring materials stacked one over the other, within a so-called heterostructure forming the interface (or heterojunction if electronic transport is specifically studied), typically interact via their lattices (e.g., strain, chemical interdiffusion, phonons) and via their charge and spin degrees of freedom (e.g., space charge layer formation, exchange bias effects). Interface-based device concepts have revolutionized the lives of humankind. 
$P-n$ junctions, metal-oxide-semiconductor fieldeffect devices, semiconducting quantum wells, and spin valves are just a few examples. As remarked by Nobel laureate Herbert Kroemer, "the interface is the device."7

In recent years, when oxide science and interface science have met, a vast realm of unexplored and ill-understood phenomena have been disclosed to solid-state scientists. Such artificial systems not only allow for combining, in a single device, the functionalities of their individual layers, they often reveal an even wider range of emergent novel properties that can be surprisingly different from those of the single building blocks.

We restrict our attention in this issue of MRS Bulletin to a broad subset of functional oxides with a perovskite structure containing two or more different cations; these are the most interesting in terms of their physical properties and, in particular, for interface science and technology because of the close-packed oxygen backbone sublattice, which is isostructural throughout the perovskite family described by the (pseudo) cubic perovskite structure, see Figure 1. These materials host a transition metal (TM) at their so-called B site that is coordinated with six atoms of the oxygen sublattices in an octahedral configuration. The TM determines most of the "electromagnetic character" of the compound with its narrow $d$-bands placed in proximity to the Fermi energy. Such "electromagnetic character" is tuned by the cation(s) populating the A site, mostly affecting the occupation of the $d$-bands and the fine details of the lattice structure (see Figure 2).

Oxide materials play a very important role in electronic devices. Nowadays, it is possible to control the electronic properties of these materials with nanoscale precision..$^{8-10}$ Many of these phenomena occur in combinations of perovskite oxides that are lattice-matched to within a few percent of one another. This enables fabrication of heteroepitaxial structures, in which multiple degrees of freedom can be accessed, potentially yielding a myriad of devices with novel functionalities. ${ }^{11}$ The properties of the oxide interfaces in such heterostructures can be exploited for applications in electronic devices.

Oxide-based devices relying on specific interface properties have already been realized and employed, such as Josephson junctions, which are non-superconducting links in superconducting circuits and superconducting quantum interference devices, magnetic tunnel junctions based on manganites (oxides of $\mathrm{Mn}$ ), and ferroelectric random access memories (FeRAMS). However, a completely new class of (nanoscale) devices can be envisaged and engineered, exploiting the functional properties of oxides. Real breakthroughs in this field can be obtained by controlling and

tailoring the physical properties at the interfaces between different oxide materials. Interfaces and surfaces in such highly correlated systems offer far more application possibilities than interfaces involving only conventional metals and semiconductors, but are more complex. ${ }^{12}$ Interfaces alter the bulk electronic system, sometimes with dramatic consequences. Interfaces break the translational and rotational symmetry, induce strain and space charge build-up, alter bond distances and angels, consequently inducing relative shifts of energy levels, modifications of bandgap values, and interfacial bandbending effects. Because these correlations control the electronic behavior of the material, their modification can induce remarkable changes in the collective electronic and magnetic properties, to the extent that phase transitions are induced. The strong interplay of the crystal degrees of freedom as well as the correlated character of electronic behavior in perovskites allows for new microscopic physical mechanisms as well as new macroscopic physical properties.

We now briefly discuss the interface that is formed in the epitaxial $\mathrm{LaAlO}_{3} / \mathrm{SrTiO}_{3}$ (LAO/STO) heterostructure, see Figure 1, whose extraordinary properties were explored for the first time by Ohtomo and Hwang. ${ }^{8}$ We employ this as an 


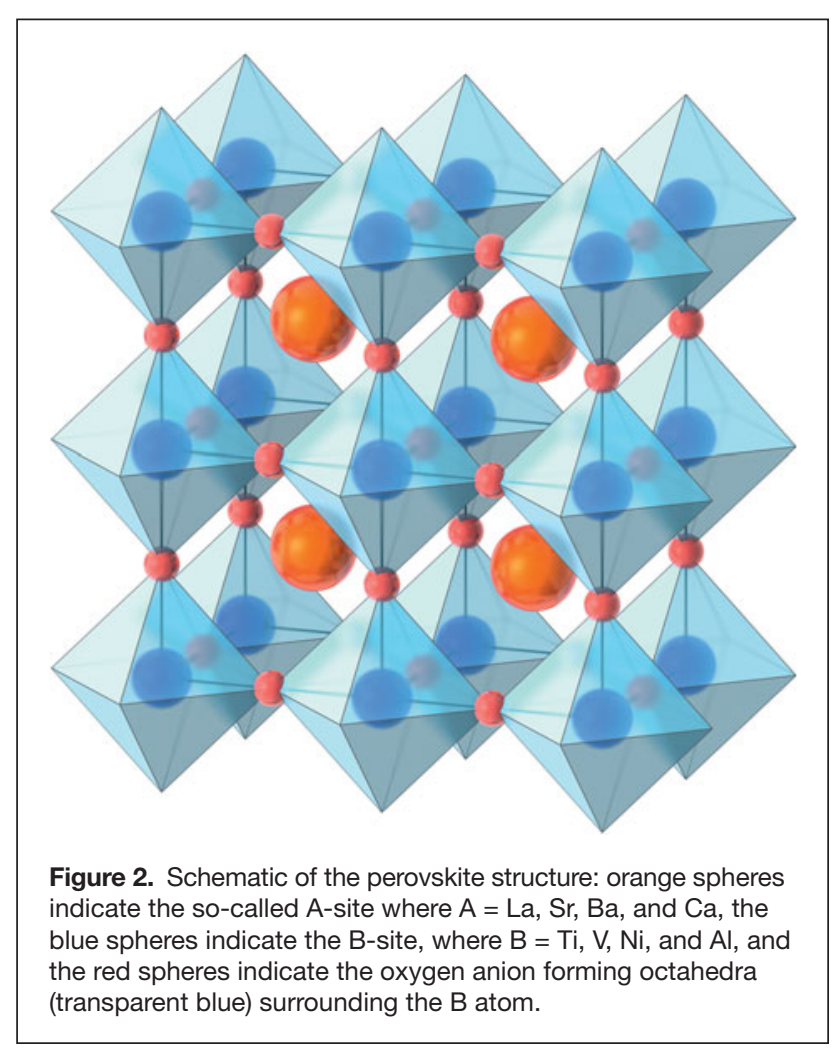

illustrative example to better explain some general peculiarities of oxide interfaces, as discussed later in this article (also see the article by Hilgenkamp in this issue).

It is instructive to describe the perovskite interfaces in terms of their constituent $\mathrm{AO}$ and $\mathrm{BO}_{2}$ layering sequence, see also Figures 1 and 2, since in high-quality heteroepitaxial growth, irrespective of the deposition method used, such a stacking sequence is preserved from one material to the other across the interface of the heterostructure. In the case of LAO/STO, the $\mathrm{SrO}$ and $\mathrm{TiO}_{2}$ layers are charge-neutral, while the $\mathrm{LaO}$ and $\mathrm{AlO}_{2}$ layers hold $\mathrm{a}+1$ and -1 charge, respectively.* Two distinct heterostructures can be obtained according to the possible stacking sequences-one containing the $\mathrm{LaO}: \mathrm{TiO}_{2}$ interface and one containing the $\mathrm{AlO}_{2}: \mathrm{SrO}$ interface. It has been shown that the so-called " $n$-type" $\mathrm{LaO}: \mathrm{TiO}_{2}$ interface becomes conducting, presumably due to electron-transfer from the $\mathrm{LaAlO}_{3}$ top layer into the formerly empty $3 \mathrm{~d} \mathrm{Ti}^{4+}$ states across the interface, a phenomenon called "electronic reconstruction" (ER). ${ }^{8}$ The ER reduces the electrostatic energy of the heterostructure by screening the diverging electrostatic potential (so-called "polar catastrophe") related to the $\mathrm{LaAlO}_{3}$ polar surfaces. As a consequence of ER, the $n$-type conducting interfaces develop a two-dimensional electron gas (2DEG) analogous to those well known in semiconductors, which find

\footnotetext{
* We are assuming here that the overall layer charge equals the sum of the ionic valences, a drastic approximation that allows a fairly truthful description of the electrostatics of this system.
}

applications in optoelectronic, high-power RF and magnetoelectronic devices, for example.

\section{What makes oxide interfaces special?}

What are the ingredients that make oxide interface science so exciting? Providing an elegant, simple answer to this question is impossible, since the factors involved are complex and manyfold. Rather, we will address a number of arguments, partially interrelated to each other, each of them contributing to making oxide interfaces unique in solid-state science: (1) availability of highly perfect epitaxial heterostructures; (2) complexity of the phase diagram and energy landscape, mostly related to electron correlations; (3) effect of multiple broken symmetries and macroscopic order parameters; and (4) complexity of defect chemistry. We will keep a special focus on the LAO/STO interface in order to provide case-by-case practical examples that better illustrate our arguments.

\section{Epitaxial heterostructures}

The first crucial prerequisite for oxide-interface-based science and technology is, trivially, the availability of a successful fabrication technology. It is a lucky case that all materials exhibiting the extraordinary previously mentioned functionalities are so similar in their chemistry and crystal structure to make the growth of epitaxial heterostructures possible. Film growers have been striving for over two decades to achieve atomic level control of the growth process.

The application of epitaxial oxide thin films in devices relying on multilayer technology requires (atomically) smooth film surfaces and interfaces. The fabrication of epitaxial complex oxide thin films involves deposition as well as subsequent growth. Here, growth implies the incorporation of adsorbed atoms (adatoms) at the film surface. Most frequently, physical vapor deposition techniques, such as sputter deposition, molecular beam epitaxy, and pulsed laser deposition (PLD), are used for the fabrication of oxide thin films.

Two further technological ingredients have played a crucial role in achieving the present level of control of the growth process: (1) control of substrate termination (i.e., the outermost atomic plane of a crystal that was first achieved for $\mathrm{SrTiO}_{3}{ }^{14,15}$ and then for other substrate materials); ${ }^{16}$ and (2) the application of real-time growth monitoring techniques, the first of which was reflection high-energy electron diffraction, in particular when adapted to the high pressure growth conditions typical of PLD growth. ${ }^{17}$ The introduction of other realtime deposition and growth monitoring techniques such as time-gated fast laser plume photography/spectroscopy ${ }^{18,19}$ and real-time $\mathrm{x}$-ray diffraction ${ }^{20,21}$ hold promise to further increase control of the growth process, probably the most delicate in the fabrication of oxide-based devices.

The case of the LAO/STO heterostructure clearly shows how much the perfect control of the atomic stacking during growth and interface science are intimately related. The arguments for explaining the formation of a 2DEG at the STO side of the interface in terms of ER strictly rely on the assumption 
that the ideal $n$-type stacking sequence described previously (also based on perfect control of the atomic $\mathrm{SrTiO}_{3}$ termination) is realized with a high degree of crystal perfection in real samples, and that the number of point defects able to screen the LAO built-in potential and to provide extra charge carriers is negligible.

\section{Phase diagrams and electron correlations}

The physics of most TM oxide systems is dominated by the competition between a fragile metallic state and electron correlations. The metallic state is due to partial overlapping of substantially localized atomic $d$ orbitals, forming relatively flat bands whose kinetic energy is further reduced in 2D systems due to the lack of dispersion along the out-of-plane direction. The correlations largely affect the charge-related degrees of freedom (often leading to so-called Mott insulators at half filling where electron-electron Coulomb repulsions prevent electrons from moving freely through the lattice), and they entangle the spin and orbital degrees of freedom in a complex manner as well. Strong coupling of the electrons with static or dynamic lattice distortion further adds to the complexity of the system. The ground states of these systems, as determined by a series of competing factors, are often highly diverse in their electromagnetic properties and still almost degenerate in energy. Minor variations in the state variables, induced by the presence of the interfaces, might induce the emergence of otherwise hidden ground states with totally novel properties.

The formation of a $2 \mathrm{D}$ electron system in $\mathrm{LAO} / \mathrm{STO}$ is an example of emergent novel properties. Electron correlations are believed to play a crucial role in determining the physics of this system that has been defined as a " $2 \mathrm{D}$ electron liquid," 22 where electrons interact and can no longer be viewed as free electrons without interactions, and includes in its phase diagram superconductivity, ${ }^{23}$ magnetism ${ }^{24}$ (also coexisting with superconductivity itself), ${ }^{25-28}$ a semiconducting/ insulating state, ${ }^{29-31}$ negative compressibility, ${ }^{32}$ a high mobility metallic state exhibiting quantum transport, ${ }^{33-35}$ including nonlocal transport, ${ }^{36}$ and so-called Rashba-induced spin-orbit coupling leading to additional splitting of the $d$-bands (details can be found in Reference 37).

\section{Broken symmetries and order parameters}

Interfaces, as mentioned previously, intrinsically break inversion symmetry. A number of other order parameters, which further reduce the symmetry of the system (by breaking, for example, time reversal, gauge, point group, chiral, or rotational symmetry), dominate the physics of TM oxides. These include electric polarization, magnetic orderings of different kinds, superconductivity, chiral states, charge and orbitally ordered states, spin and charge density wave states, and collective lattice distortions from the parent perovskite structure (e.g., of ferroelectric, antiferrodistortive or JahnTeller nature, the latter two being defined as octahedral rotations and octahedral deformations, respectively). As a general rule, such ordered states tend to decay smoothly at the interface, thus influencing the abutting material by proximity. At the interface between compounds, both showing some order parameter, multiple symmetry breaking can take place, which is an ideal condition for the emergence of totally new states of matter. ${ }^{12}$

Once again, the LAO/STO interface illustrates wonderfully the tendency of oxide interfaces to create coexisting symmetry-broken macroscopically ordered states, possessing properties such as electric polarity, ${ }^{8}$ ferromagnetism, ${ }^{21-25}$ superconductivity, ${ }^{20}$ and the Rashba effect. ${ }^{34}$ The ER mechanism can be envisaged as a "proximity effect" due to the polarization of $\mathrm{LaAlO}_{3}$ decaying smoothly into the first $\mathrm{SrTiO}_{3}$ layers. $^{38}$

\section{Defect chemistry}

TM oxides have the capability to locally accommodate a large number of point defects and still preserve the highest degree of long-range order in their crystal structure. Interfaces might host a much larger number of point defects than the constituent bulk materials. Defects already existing in the bulk, such as oxygen vacancies, might form more easily at the boundary plane than in the bulk or might migrate from the bulk to the interface to minimize their energy. Furthermore, cation interdiffusion between the two abutting materials might well take place and act as a local doping in the proximity of the interface. Since defects are ubiquitous in these systems, clearly separating the effects where lattice imperfections play no role from those in which their role is crucial has been proved so far to be a grueling task, giving rise to never-ending debates. This is certainly the case for the LAO/STO interface, where La interdiffusion ${ }^{39,40}$ or oxygen vacancies ${ }^{41}$ have been suggested as a source of "conventional" chemical doping. While some recent experiments suggest that La intermixing, though presumably present to some extent, might not play a major role in the electronic properties of high-quality LAO/STO interfaces, ${ }^{35,40}$ the dependence of transport properties on the thermodynamic history of the samples suggests that the presence of oxygen vacancies might have contributed toward determining the properties of many samples studied so far. ${ }^{32,41,42}$ Such debate has resurged after the discovery of conducting interfaces fabricated with an amorphous (and therefore certainly nonpolar) overlayer grown on $\mathrm{SrTiO}_{3}$, seemingly mimicking some of the properties of crystalline LAO/STO interfaces. ${ }^{43}$ Besides the specific LAO/STO issue, selective doping induced by interface-related crystal point defects should be considered as one of the many legitimate ingredients of oxide interface science, possibly leading to important technological and fundamental breakthroughs.

\section{Examples of functional oxide interfaces}

Given the extraordinary variety of functional properties of single functional materials, the combinations of different electromagnetic properties that can meet at oxide interfaces is almost unlimited. We informally identify some major classes and quote a few prominent examples for them. 


\section{Interfaces among insulators}

Insulators are "boring" materials, exhibiting a trivial electromagnetic response. Interfaces between oxide insulators have instead shown an unprecedented diversity of properties that are of significant fundamental and technological interest. A few examples follow.

The properties of LAO/STO and its variants ${ }^{44}$ have been discussed. They seemingly share, along with ferroelectric heterostructures, the presence of spontaneous polarization in at least one of the layers. The determination of the overall electrostatic ground state of such heterostructures is therefore best addressed by analyzing the displacement vector $\mathbf{D}$, as discussed later in this issue for ferroelectric oxide superlattices (see the article by Dawber and Bousquet in this issue) and in the case of LAO/STO. ${ }^{45,46} \mathrm{As}$ an interesting example of improper ferroelectricity, the presence of different order parameters, as collective antiferrodistortive distortions (see above), may determine the polarization of oxide superlattices, such as $\mathrm{PbTiO}_{3} / \mathrm{SrTiO}_{3}$. Strong polarization enhancements are found in asymmetric three-component (or so-called "tri-color") ferroelectric superlattices, ${ }^{47,48}$ an example of which is given in Figure 3.

Superlattices between Mott insulators (see previous explanation) and standard band insulators represent an alternative route to polar-nonpolar interfaces to confine electrons in oxide quantum wells. Examples include $\mathrm{SrTiO}_{3} / \mathrm{LaTiO}_{3} / \mathrm{SrTiO}_{3}$, $\mathrm{SrTiO}_{3} / \mathrm{LaVaO}_{3} / \mathrm{SrTiO}_{3}, \mathrm{LaAlO}_{3} / \mathrm{LaVaO}_{3} / \mathrm{LaAlO}_{3}$ and $\mathrm{SrTiO}_{3} /$ $\mathrm{SrVO}_{3} / \mathrm{SrTiO}_{3}$ (see the article by Stemmer and Millis in this issue). The highest electron mobility reported so far in a $2 \mathrm{D}$ $\mathrm{SrTiO}_{3}$-based system $\left(>100,000 \mathrm{~cm}^{2} / \mathrm{Vs}\right)$ was found at the $\gamma-\mathrm{Al}_{2} \mathrm{O}_{3} / \mathrm{SrTiO}_{3}$ spinel-perovskite interface. ${ }^{49}$ The origin of the $2 \mathrm{DEG}$ has been attributed to oxygen vacancies generated by an interface redox process, but a purely electronic process cannot be excluded. Record-high mobilities achieved so far in oxide-based heterostructures were obtained instead in the nonperovskite $\mathrm{ZnO} / \mathrm{MgZnO}$ system (i.e., $700,000 \mathrm{~cm}^{2} \mathrm{~V}^{-1} \mathrm{~s}^{-1}$ ). ${ }^{50}$ A yet ill-understood combination of different effects, such

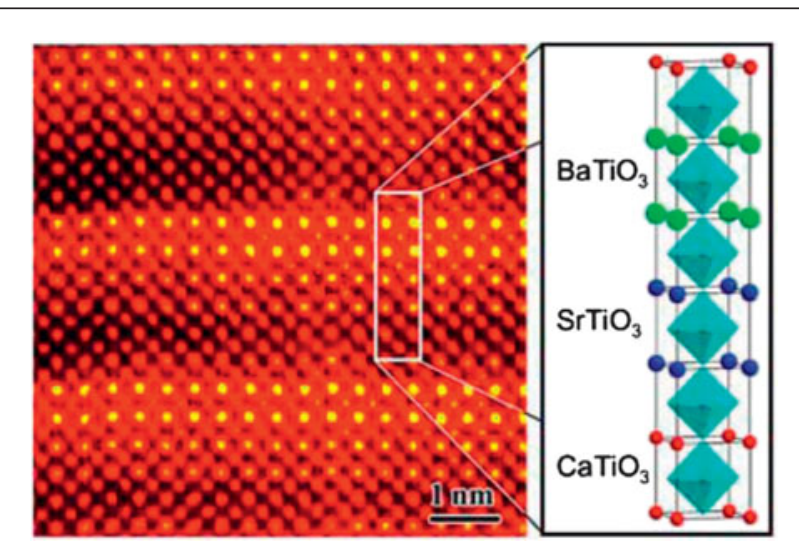

Figure 3. Cross-sectional high resolution transmission electron microscope image of an asymmetric three-component (or "tricolor") superlattice containing $(\mathrm{Ca}, \mathrm{Sr}, \mathrm{Ba}) \mathrm{TiO}_{3}{ }^{48}$ as charge transfer and space charge formation (a buildup of charge at an interface because of a difference in chemical potential), epitaxial strain, and atomic reconstructions, has been proven to cause a colossal enhancement of ionic conductivity in $\mathrm{SrTiO}_{3} / \mathrm{YSZ} / \mathrm{SrTiO}_{3}$ heterostructures (where YSZ stands for $\left.\left(\mathrm{Y}_{2} \mathrm{O}_{3}\right)_{x}\left(\mathrm{ZrO}_{2}\right)_{(1-x)}\right)$ (see also the Leon et al. article in this issue).

\section{Interfaces including metallic/superconducting/ half-metallic layers}

These interfaces differ from the previous ones described due to the presence of carriers at the Fermi level in at least one of the constituent materials. The metallic, superconducting, or ferromagnetic properties are nevertheless further modified by the interfaces and may even vanish due to size effects when ultrathin films are sandwiched in the heterostructures.

Tunneling magnetoresistence (TMR) and tunneling electroresistance (TER) junctions present spectacular hysteretic effects that can be employed in memory applications. MgObased TMR junctions with magnetic metal electrodes, such as $\mathrm{CoFeB} / \mathrm{MgO} / \mathrm{CoFeB},{ }^{51}$ represent the state of the art of TMR technology to be employed for spin-valve-based read-heads and magnetic RAMs. All-perovskite TMR junctions such as $(\mathrm{La}, \mathrm{Sr}) \mathrm{MnO}_{3} / \mathrm{SrTiO}_{3} /(\mathrm{La}, \mathrm{Sr}) \mathrm{MnO}_{3}(\mathrm{LSMO} / \mathrm{STO} / \mathrm{LSMO})$ exhibiting record-high low-temperature MR values ${ }^{52}$ unfortunately show negligible room-temperature effects. A much greater promise in the field of perovskite heterostructure technology is shown by $(\mathrm{La}, \mathrm{Sr}) \mathrm{MnO}_{3} / \mathrm{BaTiO}_{3} /(\mathrm{La}, \mathrm{Sr}) \mathrm{MnO}_{3}$ junctions, which yield a giant tunnel electroresistance value, to be potentially employed for nondestructive readout of ferroelectric states. ${ }^{53}$ Interfaces of LSMO and STO have been engineered to compensate for the existing polar discontinuity, ${ }^{54}$ see Figure 4, improving the magnetism near the interfaces.

\section{In this issue}

The articles authored by the contributors of this issue provide a wide overview of the restless and ever-surprising field of functional oxide interfaces and of the impact these systems are expected to have on future solid-state technologies. Hilgenkamp addresses in detail the LAO/STO system that already provided a thread to this introductory article. He shows that this stunning two-dimensional system exhibits a plethora of unexpected properties, including high mobility, quantum transport, charge and orbital reconstruction, Rashba-induced spin orbit coupling, and phase transitions with multiple order parameters, such as superconductivity and magnetism. Stemmer and Millis explore what is known and conjectured about confined electronic states in oxide quantum wells. Unlike the LAO/STO case, the heterostructures considered in this article are typically based on a band insulator and a Mott insulator. Different routes to control strongly correlated materials using narrow quantum wells and interfacial proximity effects are defined and addressed. Dawber and Bousquet focus on ferroelectric-based superlattices, analyzing the differences between the case of ferroelectric-dielectric heterostructures, 

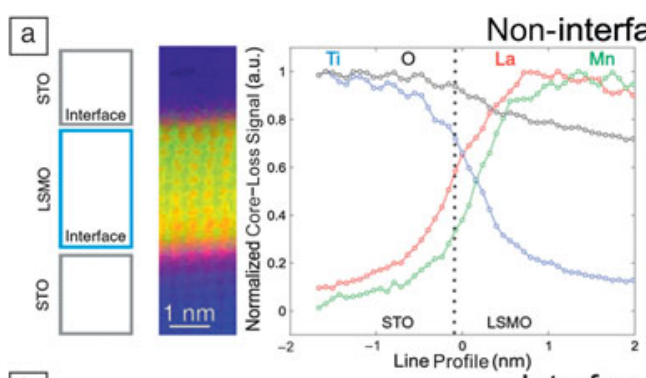

Interface
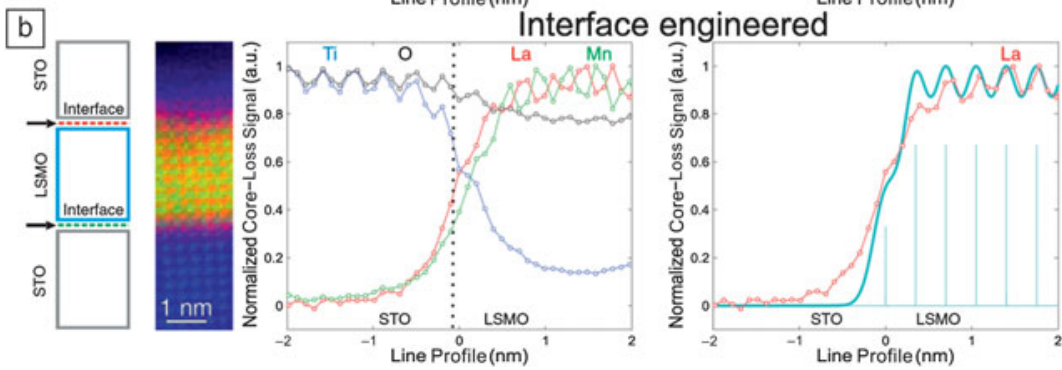

Figure 4. Electron energy loss spectroscopy analysis of the LSMO/STO heterostructures $(n=10)$ for (a) non-interface engineered and (b) interface engineered samples. Left: Quantitative color map together with a schematic of the sample structure showing the STO (gray), the LSMO (blue), and the sub-unit cell La $0.33 \mathrm{Sr} 0.67 \mathrm{O}$ layers (red and green). Middle: Normalized core-loss signals for La M 4,5 (red), Mn L 2,3 (green), Ti L 2,3 (blue), and O K (black) edges. Right: Comparison with a weighted Gaussian model (light blue) where the sticks indicate the La occupancies used in the model. For the non-interface engineered sample, clear La (red) diffusion into the STO can be observed, while no La diffusion is present for the interface engineered sample. ${ }^{54}$

ferroelectric-metal heterostructures, and others. By recognizing electric displacement as the fundamental variable to describe systems in which the spontaneous polarization is discontinuous, the distribution of polarization and surface charges in the superlattice is addressed. They show that in the case of ferroelectric-dielectric multilayers, different order parameters can set in, giving rise to improper ferroelectricity. Coey at al. analyze magnetism in heterostructures containing a magnetic layer, in films of oxides containing no evident magnetic doping, and in interfaces between non-magnetic films where magnetism appears as a novel emergent property. Opportunities to design, create, and exploit exotic magnetic oxide devices with novel functionality are discussed. Leon et al. switch the debate toward the recently born field of nano-ionics, expected to yield large improvements in the performance of oxide-based energy generation and storage. They describe the extraordinary ion-conductivity enhancement found in strained oxide heterostructures and discuss the different scenarios that have been proposed to account for such an effect.

\section{Outlook: Recent and future trends}

Perovskite $\left(\mathrm{ABO}_{3}\right)$ oxides, interfaces, and epitaxial heterostructures of these oxides exhibit exceptional physical properties, providing the basis for novel concepts of oxideelectronic devices. For a given parent compound, a rich phase diagram is colored-in by substitution of the A or B cations, and/or a change in the oxygen stoichiometry. The ionic character of the chemical bonds and the consequent possibility of electronic reconstruction often render interfaces in these materials strongly electronically active. These remarkable interface characteristics have increased interest in these materials, especially for conducting interfaces and artificial multiferroics.

Given the richness of the physical properties already found as well as the enormous parameter space that is available in the oxide system, we can be sure that we have not exhausted all possibilities. A clear trend has emerged in the search for novel properties though; on the one hand, researchers more and more focus on structural and chemical perfection of their samples (both through synthesis as well as characterization), and on the other hand, better physical modeling is becoming available. Both developments allow groups to study structureproperty relationships on a level that is unprecedented but necessary given the complexity of the materials. Who knows, for example, the unsettled issue of high $T_{\mathrm{c}}$ superconductivity might be resolved in an interface system.

Finally, inspired by the rather typical (001) perovskite orientation, orientations other than (001) are being investigated, leading to surprising results. ${ }^{55}$ In addition, crystal structures other than perovskites (e.g., spinels), ${ }^{48} \mathrm{TM}$ other than $3 d-4 d$ ones (e.g., $5 d$, Iridates), ${ }^{6,56}$ interfaces for redox resistive switching (typically not all-perovskite), ${ }^{57}$ and $\mathrm{MgO}$-based magnetic tunnel junction interfaces (non-perovskites, metal electrodes) are all being investigated. ${ }^{58}$ It is our strong conviction that both in the short term as well as the long term, oxides and their interfaces will play a prominent role in many applications.

\section{References}

1. International Technology Roadmap for Semiconductors (2011); http://www. itrs.net/Links/2011ITRS/2011Chapters/2011ERM.pdf.

2. J. Wang, J.B. Neaton, H. Zheng, V. Nagarajan, S.B. Ogale, B. Liu, D. Viehland, V. Vaithyanathan, D.G. Schlom, U.V. Waghmare, N.A. Spaldin, K.M. Rabe, M. Wuttig, R. Ramesh, Science 299 (5613), 1719 (2003).

3. N.A. Spaldin, R. Ramesh, MRS Bull. 33 (11), 1047 (2008).

4. A. Tebano, E. Fabbri, D. Pergolesi, G. Balestrino, E. Traversa, ACS Nano 6 (2), 1278 (2012).

5. M.Z. Hasan, C.L. Kane, Rev. Mod. Phys. 82, 3045 (2010).

6. X. Wan, A.M. Turner, A. Vishwanath, S.Y. Savrasov, Phys. Rev. B 83, 205101 (2011).

7. H. Kroemer, Rev. Mod. Phys. 72, 783 (2001).

8. A. Ohtomo, H.Y. Hwang, Nature 427, 423 (2004).

9. D.A. Muller, N. Nakagawa, A. Ohtomo, J.L. Grazul, H.Y. Hwang, Nature $\mathbf{4 3 0}$, 657 (2004).

10. J. Mannhart, D.G. Schlom, Nature 430, 620 (2004).

11. G. Rijnders, D.H.A. Blank, Nature 433, 369 (2005).

12. H.Y. Hwang, Y. Iwasa, M. Kawasaki, B. Keimer, N. Nagaosa, Y. Tokura, Nat. Mater. 11, 103 (2012).

13. Science 318 (5858), 1844 (2007).

14. M. Kawasaki, K. Takahashi, T. Maeda, R. Tsuchiya, M. Shinohara, O. Ishiyama, T. Yonezawa, M. Yoshimoto, H. Koinuma, Science 266 (5190), 1540 (1994).

15. G. Koster, B.L. Kropman, G.J.H.M. Rijnders, D.H.A. Blank, H. Rogalla, Appl. Phys. Lett. 73 (20), 2920 (1998).

16. J.E. Kleibeuker, G. Koster, W. Siemons, D. Dubbink, B. Kuiper, J.L. Blok, C.-H. Yang, J. Ravichandran, R. Ramesh, J.E. ten Elshof, D.H.A. Blank, G. Rijnders, Adv. Funct. Mater. 20 (20), 3490 (2010). 
17. G.J.H.M. Rijnders, G. Koster, D.H.A. Blank, H. Rogalla, Appl. Phys. Lett. 70 (14), 1888 (1997)

18. C. Aruta, S. Amoruso, R. Bruzzese, X. Wang, D. Maccariello, F. Miletto Granozio, U. Scotti di Uccio, Appl. Phys. Lett. 97, 252105 (2010)

19. A. Sambri, S. Amoruso, X. Wang, M. Radovic, F. Miletto Granozio, R. Bruzzese, Appl. Phys. Lett. 91, 151501 (2007).

20. V. Vonk, S. Konings, L. Barthe, B. Gorges, H. Graafsma, J. Synchrotron Radiat. $12(6), 833(2005)$.

21. G. Eres, J.Z. Tischler, M. Yoon, B.C. Larson, C.M. Rouleau, D.H. Lowndes, P. Zschack, Appl. Phys. Lett. 80 (18), 3379 (2002).

22. M. Breitschaft, V. Tinkl, N. Pavlenko, S. Paetel, C. Richter, J.R. Kirtley, Y.C. Liao, G. Hammerl, V. Eyert, T. Kopp, J. Mannhart, Phys. Rev. B 81, 153414 (2010).

23. N. Reyren, S. Thiel, A.D. Caviglia, L. Fitting Kourkoutis, G. Hammerl, C. Richter, C.W. Schneider, T. Kopp, A.-S. Ruetschi, D. Jaccard, M. Gabay, D. A. Muller, J.-M. Triscone, J. Mannhart, Science 317, 1196 (2007)

24. A. Brinkman, M. Huijben, M. van Zalk, J. Huijben, U. Zeitler, J.C. Maan, W.G. van der Wiel, G. Rijnders, D.H.A. Blank, H. Hilgenkamp, Nat. Mater. 6, 493 (2007). 25. Ariando, X. Wang, G. Baskaran, Z. Liu, J. Huijben, J.B. Yi, A. Annadi, A.R. Barman, A. Rusydi, S. Dhar, Y.P. Feng, J. Ding, H. Hilgenkamp, T. Venkatesan, Nat. Commun. 2 (188), (2011)

26. D.A. Dikin, M. Mehta, C.W. Bark, C.M. Folkman, C.B. Eom, V. Chandrasekhar, Phys. Rev. Lett. 107, 056802 (2011)

27. L. Li, C. Richter, J. Mannhart, R.C. Ashoori, Nat. Phys. 7, 762 (2011).

28. J.A. Bert, B. Kalisky, C. Bell, M. Kim, Y. Hikita, H.Y. Hwang, K.A. Moler, Nat. Phys. 7, 767 (2011)

29. S. Thiel, G. Hammerl, A. Schmehl, C.W. Schneider, J. Mannhart, Science 313, 1942 (2006)

30. C. Cen, S. Thiel, G. Hammerl, C.W. Schneider, K.E. Andersen, C.S. Hellberg, J. Mannhart, J. Levy, Nat. Mater. 7, 298 (2008).

31. A.D. Caviglia, S. Gariglio, N. Reyren, D. Jaccard, T. Schneider, M. Gabay, S. Thiel, G. Hammerl, J. Mannhart, J.-M. Triscone, Nature 456, 624 (2008).

32. L. Li, C. Richter, S. Paetel, T. Kopp, J. Mannhart, R.C. Ashoori, Science 332 (6031), 825 (2011).

33. M.B. Shalom, A. Ron, A. Palevski, Y. Dagan, Phys. Rev. Lett. 105, 206401 (2010)

34. A.D. Caviglia, S. Gariglio, C. Cancellieri, B. Sacépé, A. Fête, N. Reyren, M. Gabay, A.F. Morpurgo, J.-M. Triscone, Phys. Rev. Lett. 105, 236802 (2010).

35. M. Huijben, G. Koster, M.K. Kruize, S. Wenderich, J. Verbeeck, S. Bals, E. Slooten, B. Shi, H.J.A. Molegraaf, J.E. Kleibeuker, S. van Aert, J.B. Goedkoop, A. Brinkman, D.H.A. Blank, M.S. Golden, G. van Tendeloo, H. Hilgenkamp, G. Rijnders, Adv. Funct. Mater. (2013), doi:10.1002/adfm.201203355.

36. G. Cheng, J.P. Veazey, P. Irvin, C. Cen, D.F. Bogorin, F. Bi, M. Huang, S. Lu, C.-W. Bark, S. Ryu, K.-H. Cho, C.-B. Eom, J. Levy, Phys. Rev. X 3, 011021 (2013).

37. A.D. Caviglia, M. Gabay, S. Gariglio, N. Reyren, C. Cancellieri, J.-M. Triscone, Phys. Rev. Lett. 104, 126803 (2010).

38. C. Cantoni, J. Gazquez, F. Miletto Granozio, M.P. Oxley, M. Varela, A.R. Lupini, S.J. Pennycook, C. Aruta, U. Scotti di Uccio, P. Perna, D. Maccariello, Adv. Mater. 24, 3952 (2012).

39. S.A. Chambers, Surf. Sci. 605, 1133 (2011).

40. M.P. Warusawithana, C. Richter, J.A. Mundy, P. Roy, J. Ludwig, S. Paetel, T. Heeg, A.A. Pawlicki, L.F. Kourkoutis, M. Zheng, M. Lee, B. Mulcahy, W. Zander, Y. Zhu, J. Schubert, J.N. Eckstein, D.A. Muller, C. Stephen Hellberg, J. Mannhart, D.G. Schlom, Nat. Commun. 4 (2351), (2013).

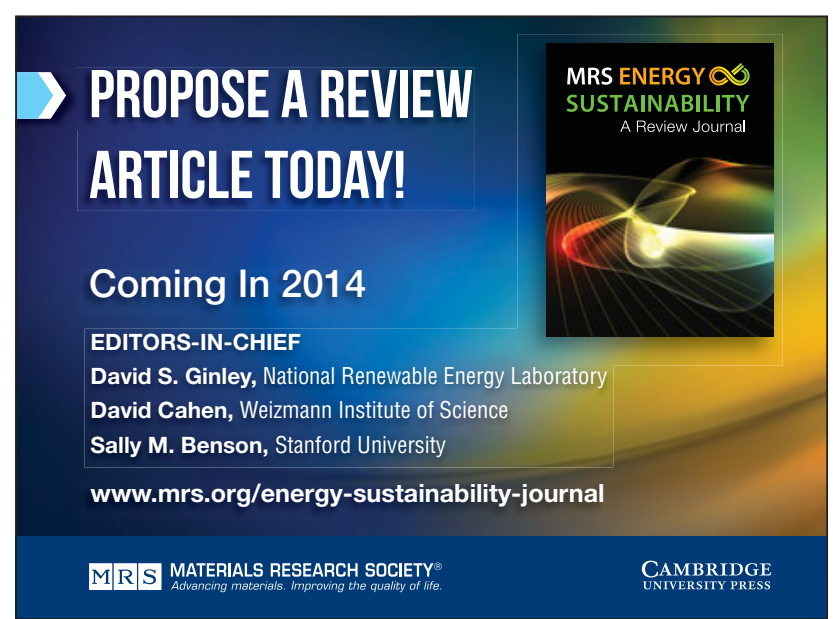

41. W. Siemons, G. Koster, H. Yamamoto, W.A. Harrison, G. Lucovsky, T.H. Geballe, D.H.A. Blank, M.R. Beasley, Phys. Rev. Lett. 98, 196802 (2007)

42. M. Basletic, J.-L. Maurice, C. Carrétéro, G. Herranz, O. Copie, M. Bibes, É. Jacquet, K. Bouzehouane, S. Fusil, A. Barthélémy, Nat. Mater. 7, 621 (2008). 43. Y. Chen, N. Pryds, J.E. Kleibeuker, G. Koster, J. Sun, E. Stamate, B. Shen, G. Rijnders, S. Linderoth, Nano Lett. 11 (9), 3774 (2011).

44. E. Di Gennaro, U. Scotti di Uccio, C. Aruta, C. Cantoni, A. Gadaleta, A.R. Lupini, D. Maccariello, D. Marré, I. Pallecchi, D. Paparo, P. Perna, M. Riaz, F. Miletto Granozio, Adv. Opt. Mater. (2013), doi:10.1002/adom.201300150. 45. M. Stengel, D. Vanderbilt, Phys. Rev. B 80, 241103(R) (2009). 46. M. Stengel, Phys. Rev. Lett. 106, 136803 (2011).

47. M.P. Warusawithana, E.V. Colla, J.N. Eckstein, M.B. Weissman, Phys. Rev Lett. 90, 030802 (2003).

48. H.N. Lee, H.M. Christen, M.F. Chisholm, C.M. Rouleau, D.H. Lowndes, Nature 433, 395 (2005).

49. Y.Z. Chen, N. Bovet, F. Trier, D.V. Christensen, F.M. Qu, N.H. Andersen, T. Kasama, W. Zhang, R. Giraud, J. Dufouleur, T.S. Jespersen, J.R. Sun, A. Smith, J. Nygård, L. Lu, B. Büchner, B.G. Shen, S. Linderoth, N. Pryds, Nat. Commun. 4 (1371) (2013), doi:10.1038/ncomms2394.

50. Y. Kozuka, S. Teraoka, J. Falson, A. Oiwa, A. Tsukazaki, S. Tarucha, M. Kawasaki, Phys. Rev. B 87, 205411 (2013).

51. S. Ikeda, J. Hayakawa, Y. Ashizawa, Y.M. Lee, K. Miura, H. Hasegawa M. Tsunoda, F. Matsukura, H. Ohno, Appl. Phys. Lett. 93 (8), 082508 (2008).

52. M. Bowen, M. Bibes, A. Barthelemy, J.P. Contour, A. Anane, Y. Lemaître, A. Fert, Appl. Phys. Lett. 82 (2), 233 (2003).

53. V. Garcia, S. Fusill, K. Bouzehouane, S. Enouz-Vedrenne, N.D. Mathur, A. Barthélémy, M. Bibes, Nature 460, 81 (2009).

54. H. Boschker, J. Verbeeck, R. Egoavil, S. Bals, G. van Tendeloo, M. Huijben, E.P. Houwman, G. Koster, D.H.A. Blank, G. Rijnders, Adv. Funct. Mater. 22 (11), 2235 (2012)

55. H. Boschker, J. Kautz, E.P. Houwman, W. Siemons, D.H.A. Blank, M. Huijben G. Koster, A. Vailionis, G. Rijnders, Phys. Rev. Lett. 109 (15), 157207 (2012). 56. D. Pesin, L. Balents, Nat. Phys. 6, 376 (2010).

57. R. Waser, R. Dittmann, G. Staikov, K. Szot, Adv. Mater. 21, 2632 (2009).

58. H. Kubota, A. Fukushima, K. Yakushiji, T. Nagahama, S. Yuasa, K. Ando, H. Maehara, Y. Nagamine, K. Tsunekawa, D.D. Djayaprawira, N. Watanabe Y. Suzuki, Nat. Phys. 4, 37 (2008)
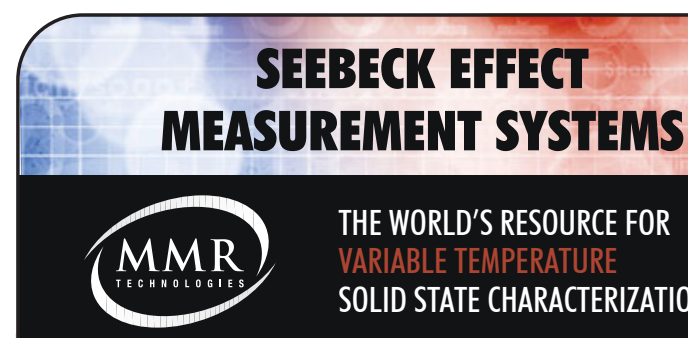

THE WORLD'S RESOURCE FOR VARIABLE TEMPERATURE SOLID STATE CHARACTERIZATION

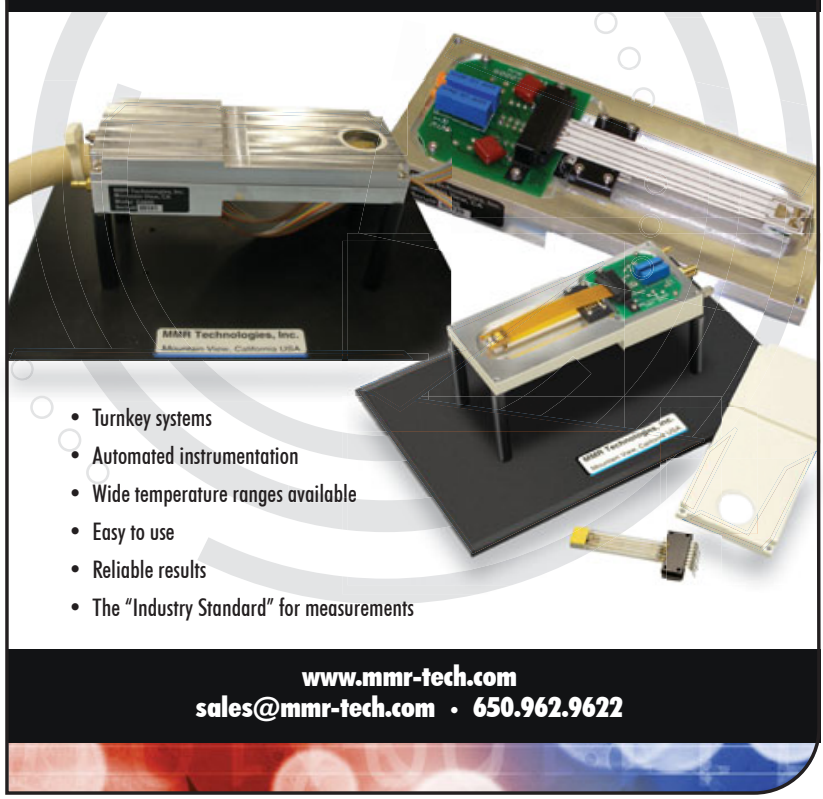

